\title{
Anomalous Higgs Couplings at the LHeC
}

\author{
A. Senol* \\ Kastamonu University, Department of Physics, 37100, Kastamonu, Turkey and \\ Abant Izzet Baysal University, Department of Physics, 14280, Bolu, Turkey
}

\begin{abstract}
The discovery of Higgs boson plays a crucial role in understanding the electroweak symmetry breaking sector. From now on, solving the dynamics of this sector needs precision measurements of the couplings of the Higgs boson to the standard model particles. In this work, we investigate the constrains on the anomalous $H W W$ and $H W W \gamma$ couplings, described by the dimension-six operators in the effective Lagrangian, in a high energy envisaged ep collider which is called Large Hadron electron Collider (LHeC). We obtained the $95 \%$ confidence level limits on the couplings of anomalous $H W W$ and $H W W \gamma$ vertex, with the design luminosity of $10 \mathrm{fb}^{-1}$ and electron beam energy of $140 \mathrm{GeV}$, through $e p \rightarrow \nu H+X, \gamma p \rightarrow W H+X$ and $e \gamma \rightarrow W H \nu$ processes by considering the new physics energy scale to be $\Lambda=1 \mathrm{TeV}$. The sensitivity of the LHeC to the new physics scale is also briefly discussed.
\end{abstract}

PACS numbers: 12.60.Fr, 14.80.Cp

*Electronic address: asenol@kastamonu.edu.tr 


\section{INTRODUCTION}

After the discovery of a new boson being compatible with Standard Model(SM) Higgs boson production and decay by ATLAS [1] and CMS [2] Collaborations at the Large Hadron Collider (LHC), the Electroweak Symmetry Breaking (EWSB) mechanism was verified experimentally leading to open up a gateway for new research field in particle physics. Now, the constraints on couplings of Higgs boson with the SM particles need to be reconsidered due to the fact that the precision measurements of its couplings will give us detailed information on EWSB of the SM and beyond. Therefore, we focus on anomalous couplings of $H W W$ and $H W W \gamma$ vertex in ep collision where some advantages over the LHC for precision measurements such as: the ability to separate backward scattering and forward scattering due to characteristic ep kinematics and an anomalous $H W W$ vertex will be free from possible contaminations of other Higgs boson-electroweak vector boson couplings.

Recently, there has been a new ep collider project, the Large Hadron Electron Collider (LHeC) [3], in which a newly built electron beam of $60 \mathrm{GeV}$, to possibly $140 \mathrm{GeV}$, energy collides with the intense hadron beams of the LHC (7 TeV) and with the design luminosity of $10^{33} \mathrm{~cm}^{-2} \mathrm{~s}^{-1}$. The physics programme is purposed to a search of the energy frontier, complementing the LHC and its discovery potential for physics beyond the Standard Model.

There have been several studies for anomalous couplings of $H W W$ vertex in the literature which focus on future linear $e^{+} e^{-}$collider [4-10] and its $e \gamma[11,12]$ and $\gamma \gamma$ [13 16] modes , hadron colliders [17-25] and also ep collider [27]. In Ref. [27], the constrains on anomalous $\mathrm{CP}$-conserving and CP-violating couplings of $H W W$ vertex coming from dimension-five operators in the effective Lagrangian are studied. Furthermore, we will analyze the anomalous couplings of $H W W$ and $H W W \gamma$ vertex coming from dimension-six operators in the effective Lagrangian.

The Higgs-vector boson vertices are uniquely assigned in the SM. In some models deviations from these vertices appear, such as non-pointlike character of boson and through interactions beyond the SM. We do not have a specific model to analyze for the effect of non-SM couplings. We investigated anomalous Higgs-vector boson couplings in a model independent way by means of effective non-renormalizable Lagrangian approach which keep 
the SM gauge group [5, 28]

$$
\mathcal{L}_{e f f}=\mathcal{L}_{S M}+\sum_{k=1}^{\infty} \frac{1}{\left(\Lambda^{2}\right)^{k}} \sum_{i} f_{i}^{(k)} Q_{i}^{d_{k}}
$$

where $d_{k}=2 k+4$ denotes the dimension of operators and $\Lambda$ is the energy scale of new interactions. We study only to complete set of the dimension- 6 operators.

In this framework, there are only two relevant operators that Higgs boson couplings to electroweak vector bosons:

$$
\frac{1}{\Lambda^{2}}\left\{\frac{1}{2} f_{\varphi} \partial_{\mu}\left(\Phi^{+} \Phi\right) \partial^{\mu}\left(\Phi^{+} \Phi\right)+f_{W W} \Phi^{+}\left(\hat{W}_{\mu \nu} \hat{W}^{\mu \nu}\right) \Phi\right\}
$$

We use the formalism of [29] in writing for $H W W$ and $H W W \gamma$ vertices in unitary gauge which follow from the effective Lagrangian (11) and (2):

$$
\left.\Gamma_{\mu \nu}^{H W W}(p, q, r)=\frac{e M_{W}}{s_{W}}\left\{\left(1-\frac{1}{4} f_{\varphi} \frac{v^{2}}{\Lambda^{2}}\right) g_{\mu \nu}+f_{W W} \frac{1}{\Lambda^{2}}\left[g_{\mu \nu}(q \cdot r)-q_{\nu} r_{\mu}\right)\right]\right\}
$$

and

$$
\Gamma_{\mu \nu \alpha}^{H W W}(p, q, r, l)=\frac{e^{2} M_{W}}{s_{W}} 2 f_{W W} \frac{1}{\Lambda^{2}}\left\{g_{\mu \nu}(q-r)_{\alpha}-q_{\nu} g_{\mu \alpha}+r_{\mu} g_{\nu \alpha}\right\}
$$

where $v=\frac{2 M_{W}}{e} s_{W}$ is the vacuum expectation value; $p, q, r$ and $l$ are the momenta of the $H, W^{+}, W^{-}$and $\gamma$ fields, respectively. $\mu, \nu$ and $\alpha$ denote the $W^{\prime}$ s and $\gamma$ fields, respectively. If the values of $f_{W W}$ and $f_{\varphi}$ are zero in $\Gamma_{\mu \nu}^{H W W}$ vertex, it corresponds to the SM vertex at tree level. The second vertex $\Gamma_{\mu \nu \alpha}^{H W W \gamma}$ does not occur in the SM at tree level. All calculations were performed by means of computer package the CalcHEP [30], after implementation of the vertices (3) and (41) with taking $\Lambda=1 \mathrm{TeV}$ and $m_{H}=125 \mathrm{GeV}$.

\section{THE CROSS SECTIONS OF $e p \rightarrow \nu H+X, \gamma p \rightarrow W H+X$ AND $e \gamma \rightarrow W H \nu$ PROCESSES}

The production mechanism for a Higgs boson in the $W W$ fusion at the LHeC is $e p \rightarrow$ $\nu H+X$ as shown in Fig. 1. This process has a single Feynman diagram involving the $H W W$ vertex. In the left panel of Fig. 2, we display the total cross sections depending on incoming electron energy for the reaction $e p \rightarrow \nu H+X$ including only anomalous $H W W$ 


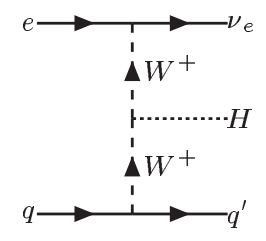

FIG. 1: Tree-level Feynman diagram for the process $e p \rightarrow \nu H+X$.

coupling with taking $f_{W W}\left(f_{\varphi}\right)=1(0) \mathrm{TeV}^{-2}, f_{\varphi}\left(f_{W W}\right)=-1(0) \mathrm{TeV}^{-2}$ and $f_{W W}=f_{\varphi}=0$ for illustration purpose by using parton distribution functions library CTEQ6L [31]. The calculated total cross sections of the $e p \rightarrow \nu H+X$ process with taking $140 \mathrm{GeV}$ of energy of incoming electron as function of the anomalous couplings $f_{W W}$ and $f_{\varphi}$ is shown in the right panel of Fig. 2, Here and henceforth, only one of the coupling parameter is kept from zero. From left panel of Fig. 2 we can see that, the only contribution from the SM part of the Eq. (3) in the case of $f_{W W}=f_{\varphi}=0$ (solid line), from both $f_{\varphi}\left(f_{W W}\right)$ coupling and SM part in the case of $f_{\varphi}=-1(0) \mathrm{TeV}^{-2}, f_{W W}=0$ (1) $\mathrm{TeV}^{-2}$ to $e p \rightarrow \nu H+X$ process. Here, we see much larger deviation from SM cross sections on the positive region of the anomalous coupling $f_{\varphi}$ due to the negative factor front the anomalous coupling $f_{\varphi}$ in the Eq. (3).
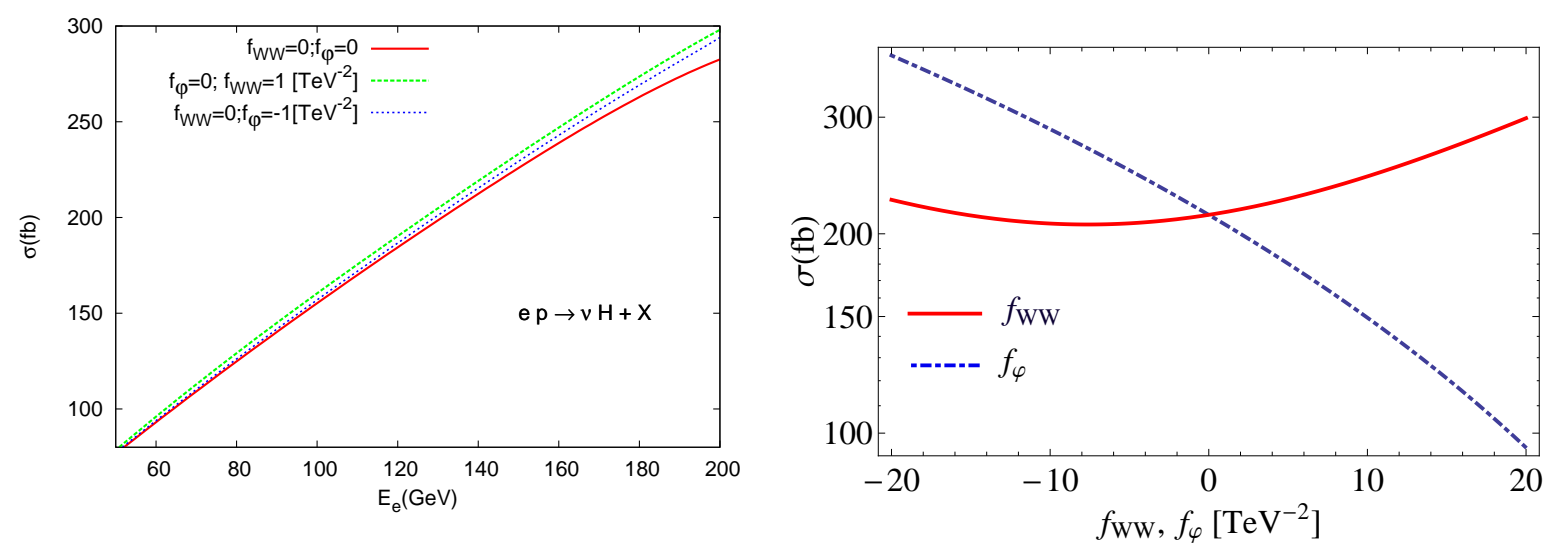

FIG. 2: The total cross sections for the process $e p \rightarrow \nu H+X$ including only anomalous $H W W$ couplings in ep collisions at the LHeC. The plot on the left displays incoming electron energy dependence with taking $m_{H}=125 \mathrm{GeV}$. The plot on the right shows dependence on anomalous couplings $f_{W W}$ (solid line) and $f_{\varphi}$ (dashed line) with taking $E_{e}=140 \mathrm{GeV}$ and $m_{H}=125 \mathrm{GeV}$.

Efficient $\gamma p$ collisions can be realized with real $\gamma$, produced using Compton back scattering 

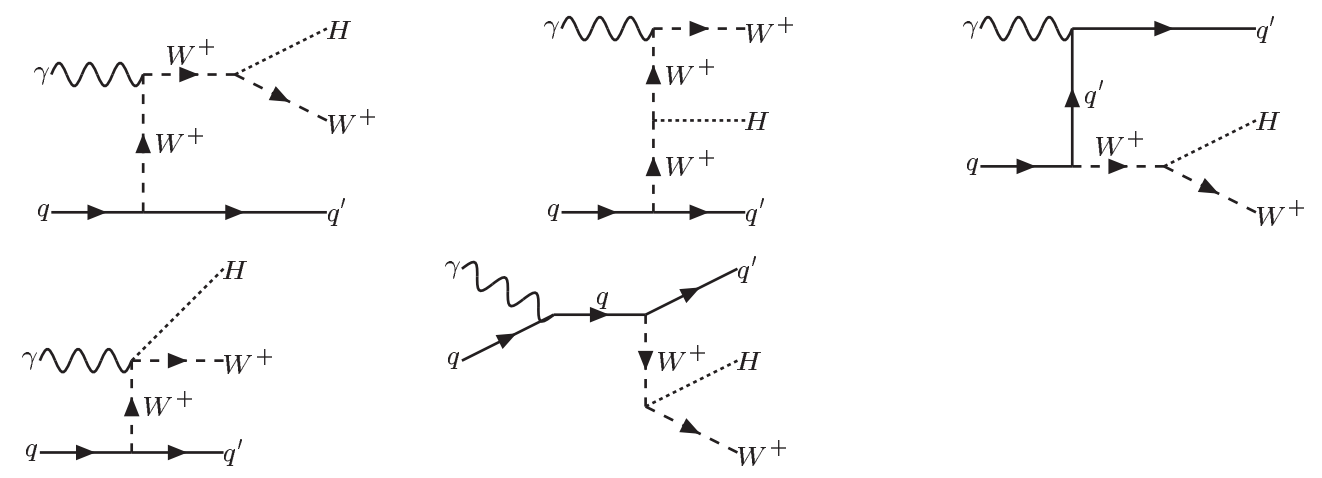

FIG. 3: Tree-level Feynman diagrams for the process $\gamma p \rightarrow W H+X$.

of laser beam off the high energy electron beam, only on the base of linac ring type ep colliders [32]. In this framework, we consider $\gamma p \rightarrow W H+X$ reaction to see the effect of both $H W W$ and $H W W \gamma$ couplings. The tree-level diagrams of the process $\gamma p \rightarrow W H+X$ are depicted in Fig. 3. We present the total cross section as function of incoming electron beam energy for this process by using the spectrum of photons scattered backward from the interaction of laser light with the high energy electron beam [33] in case $f_{W W}=f_{\varphi}=0, f_{W W}\left(f_{\varphi}\right)=1(0)$ $\mathrm{TeV}^{-2}$ and $f_{\varphi}\left(f_{W W}\right)=-1(0) \mathrm{TeV}^{-2}$ in the left panel of Fig. 4. As we can see, contribution of the $H W W \gamma$ vertex, described in Eq. (4), leads to an increase of about two orders in the cross section. Total cross sections of $\gamma p \rightarrow W H+X$ process as a functions of $f_{W W}$ and $f_{\varphi}$ is shown in the left panel of Fig. 4,

The another mode of ep colliders is $e \gamma$ option where $\gamma$ is elastic photon emission coming from proton. The equivalent photon spectrum is described by the equivalent photon approximation (EPA) [34] which embedded in CalcHEP. The $e \gamma \rightarrow W H \nu$ process in ep collision is described by tree-level diagrams in Fig. 5. These diagrams contain anomalous $H W W$ and $H W W \gamma$ couplings. In Fig. 6, we plot the total cross section depending on incoming electron energy for $f_{W W}=f_{\varphi}=0, f_{W W}\left(f_{\varphi}\right)=1(0) \mathrm{TeV}^{-2}$ and $f_{\varphi}\left(f_{W W}\right)=-1$ (0) $\mathrm{TeV}^{-2}$ (left panel) and as functions of $f_{W W}$ and $f_{\varphi}$ with taking $E_{e}=140 \mathrm{GeV}$ (right panel) by using EPA. 

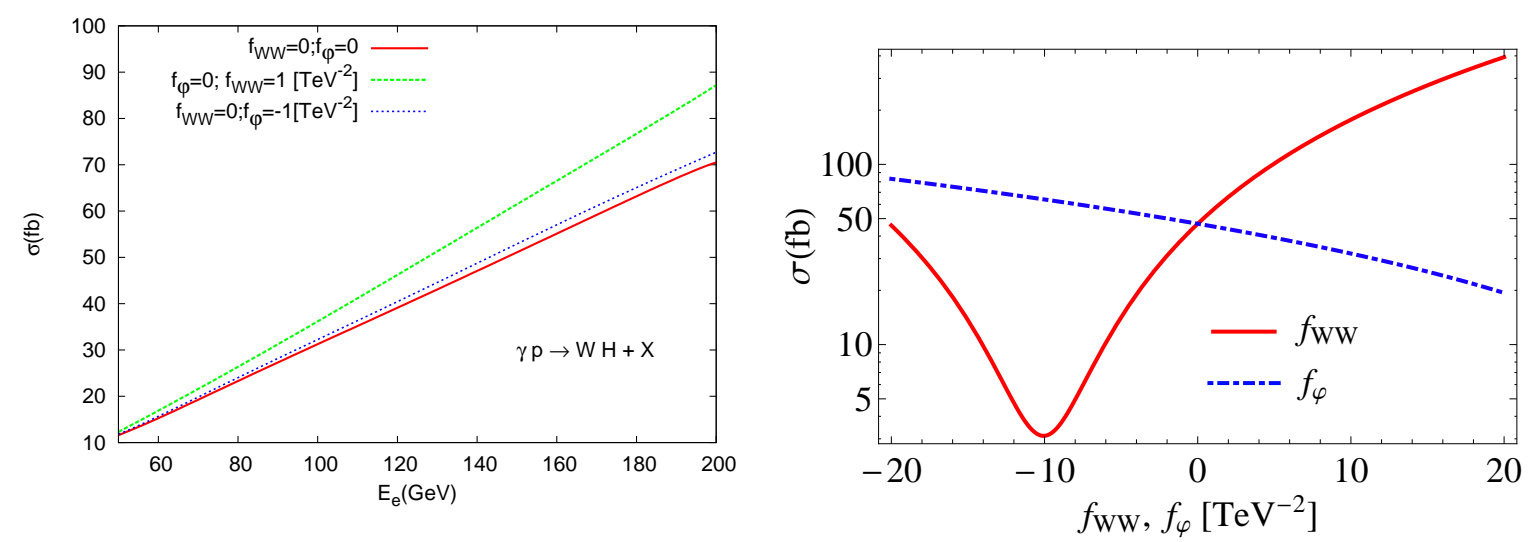

FIG. 4: The total cross sections depending on incoming electron energy for $\gamma p \rightarrow W H+X$ including anomalous $H W W$ and $H W W \gamma$ couplings in ep collisions at the LHeC.The plot on the left displays incoming electron energy dependence with taking $m_{H}=125 \mathrm{GeV}$. The plot on the right shows dependence on anomalous couplings $f_{W W}$ (solid line) and $f_{\varphi}$ (dashed line) with taking $E_{e}=140 \mathrm{GeV}$ and $m_{H}=125 \mathrm{GeV}$.
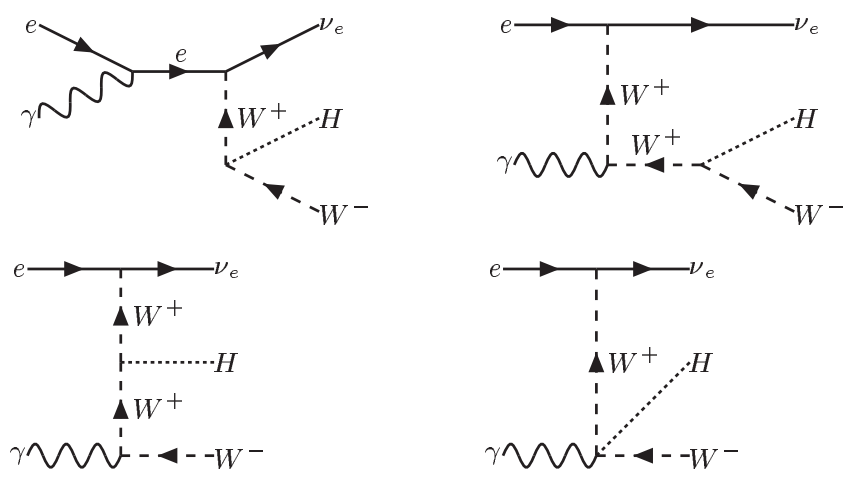

FIG. 5: Tree-level Feynman diagrams for the process $e \gamma \rightarrow W H \nu$.

\section{LIMITS ON THE ANOMALOUS HIGGS COUPLINGS}

One-parameter $\chi^{2}$ test was applied without a systematic error to obtain $95 \%$ confidence level (C.L.) on the upper limits of the $f_{\varphi}$ and $f_{W W}$. The $\chi^{2}$ function is

$$
\chi^{2}=\left(\frac{\sigma_{S M}-\sigma\left(f_{\varphi}, f_{W W}\right)}{\sigma_{S M} \delta}\right)^{2}
$$



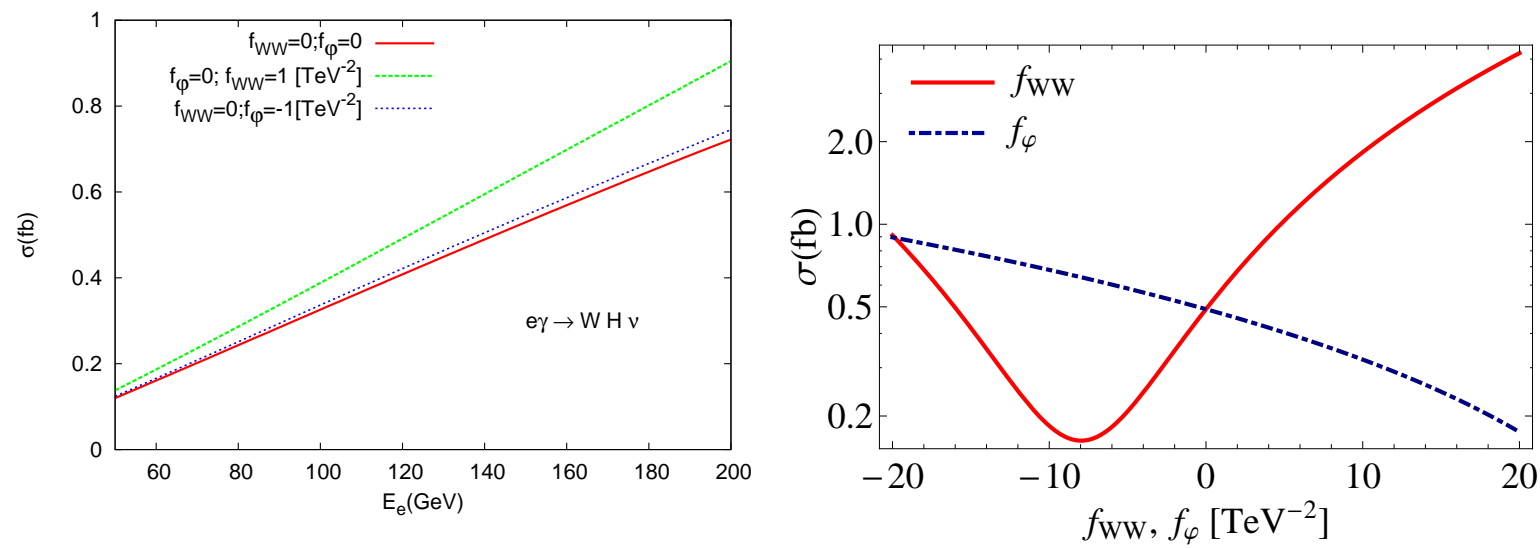

FIG. 6: The total cross sections depending on incoming electron energy for $e \gamma \rightarrow W H \nu$ including anomalous $H W W$ and $H W W \gamma$ couplings in ep collisions at the LHeC.The plot on the left displays incoming electron energy dependence with taking $m_{H}=125 \mathrm{GeV}$. The plot on the right shows dependence on anomalous couplings $f_{W W}$ (solid line) and $f_{\varphi}$ (dashed line) with taking $E_{e}=140$ $\mathrm{GeV}$ and $m_{H}=125 \mathrm{GeV}$.

where $\delta=\frac{1}{\sqrt{N}}$ is the statistical error. The number of events are given by $N=\sigma_{S M} L_{\text {int }}$ where $L_{i n t}$ is the integrated luminosity. When calculating number of events we assume all $W$ bosons decay leptonically in the final state, the dominant Higgs boson decay to $b \bar{b}$, the efficiency for b-tagging to be $\epsilon=60 \%$ and the fake rejection factors of 0.01 for light quarks. And also we applied cuts for missing transverse energy (MET) for neutrinos to be MET $>25 \mathrm{GeV}$, transverse momentum of quarks to be $p_{T}^{b, j}>30 \mathrm{GeV}$ and pseudorapidity of quarks to be $|\eta|^{b, j}<2.5$. With assuming these restrictions, we have calculated total cross sections $\sigma_{S M}=0.047 \mathrm{pb}$ for $e p \rightarrow \nu H+X, \sigma_{S M}=7.61 \times 10^{-3} \mathrm{pb}$ for $\gamma p \rightarrow H W+X$ and $\sigma_{S M}=3.72 \times 10^{-4} \mathrm{pb}$ for $e \gamma \rightarrow H W \nu$ processes.

In Fig. 7, we exhibited $\chi^{2}$ as a function of $f_{W W}$ (left panel) and $f_{\varphi}$ (right panel) through $e p \rightarrow \nu H+X, \gamma p \rightarrow W H+X$ and $e \gamma \rightarrow W H \nu$ with $\Lambda=1 \mathrm{TeV}, E_{e}=140 \mathrm{GeV}$ and design luminosity, $L=10 \mathrm{fb}^{-1}$. A distinct feature of this figure is that the limiting on anomalous couplings to see clearly at $95 \%$ C.L.. If the LHeC has collected $10 \mathrm{fb}^{-1}$ of data, the bounds on $f_{W W}$ would be $(-39.3,27.4) \mathrm{TeV}^{-2}$ for $e p \rightarrow \nu H+X,(-29.8,11.9) \mathrm{TeV}^{-2}$ for $\gamma p \rightarrow W H+X,(-31.5,11.3) \mathrm{TeV}^{-2}$ for $e \gamma \rightarrow W H \nu$ process and $f_{\varphi}$ would be $(-47.2,167.2)$ $\mathrm{TeV}^{-2}$ for $e p \rightarrow \nu H+X,(-153.9,261.2) \mathrm{TeV}^{-2}$ for $\gamma p \rightarrow W H+X,(-79.0,237.1) \mathrm{TeV}^{-2}$ for $e \gamma \rightarrow W H \nu$ at $95 \%$ C.L. While the indirect $95 \%$ C.L. constraints of the L3 collaboration 
TABLE I: Variations of $f_{W W}$ and $f_{\varphi}$ couplings with respect to $\Lambda$ at $95 \%$ C.L. for $e p \rightarrow \nu H+X$, $\gamma p \rightarrow W H+X$ and $e \gamma \rightarrow W H \nu$ processes with $E_{e}=140 \mathrm{GeV}$ and design luminosity of $10 \mathrm{fb}^{-1}$.

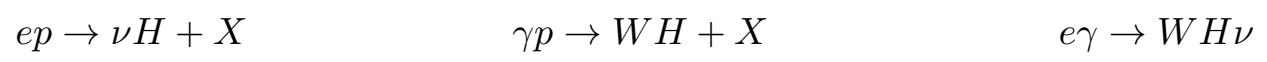

\begin{tabular}{lcccccc}
$\Lambda(\mathrm{TeV})$ & $f_{W W}$ & $f_{\varphi}$ & $f_{W W}$ & $f_{\varphi}$ & $f_{W W}$ & $f_{\varphi}$ \\
\hline 1 & $(-39.3,27.4)$ & $(-47.2,167.2)$ & $(-29.8,11.9)$ & $(-153.9,261.2)$ & $(-31.5,11.3)$ & $(-79.0,237.1)$ \\
2 & $(-44.7,37.2)$ & $(-175.7,575.3)$ & $(-117.2,48.3)$ & $(-495.1,789.2)$ & $(-129.9,43.8)$ & $(-306.9,968.9)$ \\
3 & $(-338.3,218.3)$ & $(-281.7,973.8)$ & $(-238.5,115.9)$ & $(-714.6,1004.3)$ & $(-288.4,99.7)$ & $(-634.5,2395.6)$ \\
\hline \hline
\end{tabular}

[21, 35] for $f_{W W}$ are in the interval of $(-26.84,26.84) \mathrm{TeV}^{-2}$ with taking $m_{H}=120 \mathrm{GeV}$ and $(-7.0,10) \mathrm{TeV}^{-2}$ form available data from Tevatron and LHC at 90\% C.L. [25, 26].

In Table I, we give $95 \%$ C.L. bounds of the couplings $f_{W W}$ and $f_{\varphi}$ for three different values of new physics energy scale, $\Lambda$, at $e p \rightarrow \nu H+X, \gamma p \rightarrow W H+X$ and $e \gamma \rightarrow W H \nu$ processes with the design luminosity of $10 \mathrm{fb}^{-1}$ and electron beam energy of $140 \mathrm{GeV}$. The fact that the sensitivity of coupling $f_{\varphi}$ is more rapidly decrease, compared to $f_{W W}$, when the scale of new physics increase. On the other hand, we can see a faster drop on the sensitivity of coupling $f_{W W}$ in $e p \rightarrow \nu H+X$ process, compared to $e \gamma \rightarrow W H \nu$, at $\Lambda=3 \mathrm{TeV}$.
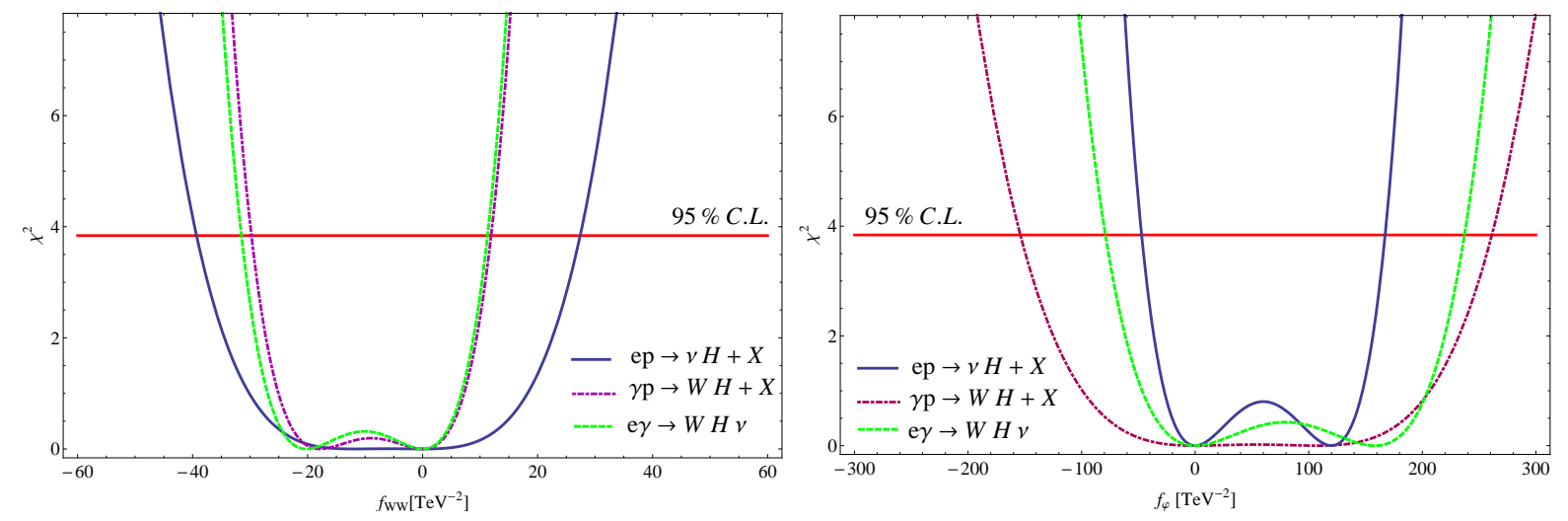

FIG. 7: $\chi^{2}$ as a function of $f_{W W}$ (left panel) and $f_{\varphi}$ (right panel) through $e p \rightarrow \nu H+X, \gamma p \rightarrow$ $W H+X$ and $e \gamma \rightarrow W H \nu$ with $E_{e}=140 \mathrm{GeV}$ and design luminosity of $10 \mathrm{fb}^{-1}$. 


\section{CONCLUSION}

In this work, we focused on couplings of $H W W$ and $H W W \gamma$ vertices to constrain deviations from the SM behavior leading the effects of dimension-six effective operators by considering the new physics energy scale to be $\Lambda=1 \mathrm{TeV}$. We have examined these effects at $e p \rightarrow \nu H+X, \gamma p \rightarrow W H+X$ and $e \gamma \rightarrow W H \nu$ processes at the LHeC to compare which can give the best limits on the anomalous couplings. Best limits on $f_{W W}$ are obtained about $(-39.3,27.4) \mathrm{TeV}^{-2}$ at $e p \rightarrow \nu H+X$ process, $(-29.8,11.9) \mathrm{TeV}^{-2}$ at $\gamma p \rightarrow W H+X$ and $(-31.5,11.3) \mathrm{TeV}^{-2}$ at $e \gamma \rightarrow W H \nu$ process and the limits on $f_{\varphi}$ are obtained about $(-42.45,7.18) \mathrm{TeV}^{-2}$ at $e p \rightarrow \nu H+X,(-153.9,261.2) \mathrm{TeV}^{-2}$ at $\gamma p \rightarrow W H+X,(-79.0,237.1)$ $\mathrm{TeV}^{-2}$ in $e \gamma \rightarrow W H \nu$ at $95 \%$ C.L. with the design luminosity value. The sensitivity on anomalous couplings, $f_{W W}$ and $f_{\varphi}$, with respect to new physics scale are investigated. It is shown that the sensitivity of $f_{\varphi}$ rapidly decrease, compared to $f_{W W}$, when the scale of new physics increase. We cannot simply compare our results on $f_{W W}$ and $f_{\varphi}$ one to one with the experimental limits obtained by various sources due to the different conventions adopted in the literature. However, the current experimental limits are of same order as our bounds. As well as, an integrated luminosity of $10 \mathrm{fb}^{-1}$ would be enough to probe small values of anomalous Higgs couplings. Nevertheless, the LHeC is a suitable platform to complement the LHC results for searching of anomalous $H W W$ and $H W W \gamma$ couplings in $e p \rightarrow \nu H+X$ process as well as $\gamma p \rightarrow W H+X$ and $e \gamma \rightarrow W H \nu$ processes.

[1] G. Aad et al. [ATLAS Collaboration], Phys. Lett. B 716, 1 (2012) arXiv:1207.7214 [hep-ex]].

[2] S. Chatrchyan et al. [CMS Collaboration], Phys. Lett. B 716, 30 (2012) arXiv:1207.7235 [hep-ex]].

[3] J. L. Abelleira Fernandez et al. [LHeC Study Group Collaboration], J. Phys. G 39, 075001 (2012) arXiv:1206.2913 [physics.acc-ph]].

[4] K. Hagiwara, S. Ishihara, R. Szalapski and D. Zeppenfeld, Phys. Rev. D 48, 2182 (1993).

[5] K. Hagiwara, R. Szalapski and D. Zeppenfeld, Phys. Lett. B 318, 155 (1993) hep-ph/9308347.

[6] G. J. Gounaris, F. M. Renard and N. D. Vlachos, Nucl. Phys. B 459, 51 (1996) hep-ph/9509316.

[7] W. Kilian, M. Kramer and P. M. Zerwas, Phys. Lett. B 381, 243 (1996) hep-ph/9603409. 
[8] S. M. Lietti, S. F. Novaes and R. Rosenfeld, Phys. Rev. D 54, 3266 (1996) hep-ph/9603343.

[9] S. S. Biswal, D. Choudhury, R. M. Godbole and Mamta, Phys. Rev. D 79, 035012 (2009) [arXiv:0809.0202 [hep-ph]].

[10] S. S. Biswal, R. M. Godbole, R. K. Singh and D. Choudhury, Phys. Rev. D 73, 035001 (2006) [Erratum-ibid. D 74, 039904 (2006)] hep-ph/0509070].

[11] I. Sahin, Phys. Rev. D 77, 115010 (2008) arXiv:0802.0293 [hep-ph]].

[12] D. Choudhury and Mamta, Phys. Rev. D 74, 115019 (2006) hep-ph/0608293.

[13] G. J. Gounaris and F. M. Renard, Z. Phys. C 69, 513 (1996) hep-ph/9505429].

[14] A. T. Banin, I. F. Ginzburg and I. P. Ivanov, Phys. Rev. D 59, 115001 (1999) hep-ph/9806515.

[15] T. Han, Y. -P. Kuang and B. Zhang, Phys. Rev. D 73, 055010 (2006) hep-ph/0512193.

[16] B. Sahin, J. Phys. G 36, 025012 (2009) arXiv:0808.0842 [hep-ph]].

[17] F. de Campos, M. C. Gonzalez-Garcia and S. F. Novaes, Phys. Rev. Lett. 79, 5210 (1997) hep-ph/9707511.

[18] M. C. Gonzalez-Garcia, Int. J. Mod. Phys. A 14, 3121 (1999) hep-ph/9902321.

[19] H. -J. He, Y. -P. Kuang, C. P. Yuan and B. Zhang, Phys. Lett. B 554, 64 (2003) hep-ph/0211229.

[20] B. Zhang, Y. -P. Kuang, H. -J. He and C. P. Yuan, Phys. Rev. D 67, 114024 (2003) hep-ph/0303048.

[21] V. Hankele, G. Klamke, D. Zeppenfeld and T. Figy, Phys. Rev. D 74, 095001 (2006) hep-ph/0609075.

[22] S. Kanemura and K. Tsumura, Eur. Phys. J. C 63, 11 (2009) arXiv:0810.0433 [hep-ph]].

[23] N. Desai, D. K. Ghosh and B. Mukhopadhyaya, Phys. Rev. D 83, 113004 (2011) arXiv:1104.3327 [hep-ph]].

[24] F. Bonnet, M. B. Gavela, T. Ota and W. Winter, Phys. Rev. D 85, 035016 (2012) arXiv:1105.5140 [hep-ph]].

[25] T. Corbett, O. J. P. Eboli, J. Gonzalez-Fraile and M. C. Gonzalez-Garcia, Phys. Rev. D 86, 075013 (2012) arXiv:1207.1344 [hep-ph]].

[26] T. Corbett, O. J. P. Eboli, J. Gonzalez-Fraile and M. C. Gonzalez-Garcia, Phys. Rev. D 87, 015022 (2013) arXiv:1211.4580 [hep-ph]].

[27] S. S. Biswal, R. M. Godbole, B. Mellado and S. Raychaudhuri, arXiv:1203.6285 [hep-ph]. 
[28] W. Buchmuller and D. Wyler, Nucl. Phys. B 268, 621 (1986).

[29] E. Boos, A. Pukhov, M. Sachwitz and H. J. Schreiber, Z. Phys. C 75, 237 (1997) hep-ph/9610424.

[30] A. Belyaev, N. D. Christensen and A. Pukhov, arXiv:1207.6082 [hep-ph].

[31] J. Pumplin, D. R. Stump, J. Huston, H. L. Lai, P. M. Nadolsky and W. K. Tung, JHEP 0207, 012 (2002) hep-ph/0201195.

[32] U. Kaya, S. Sultansoy and G. Unel, arXiv:1211.5061 [hep-ph].

[33] I. F. Ginzburg, G. L. Kotkin, V. G. Serbo and V. I. Telnov, Nucl. Instrum. Meth. 205, 47 (1983).

[34] V. M. Budnev, I. F. Ginzburg, G. V. Meledin and V. G. Serbo, Phys. Rept. 15, 181 (1975).

[35] P. Achard et al. [L3 Collaboration], Phys. Lett. B 589, 89 (2004) hep-ex/0403037. 